\title{
Suzanne Smitz-De Smet

\author{
Abstracts of the 16th Annual Congress \\ of the European Society \\ of Intensive Care Medicine, \\ Amsterdam, Netherlands
}

Published online: 3 February 2004

(C) Springer-Verlag 2004

The online versions of the original articles can be found at: http://dx.doi.org/10.1007/s00134-003-1971-0, http://dx.doi.org/10.1007/s00134-003-1973-y, http://dx.doi.org/10.1007/s00134-003-1974-x

S. Smitz-De Smet (

European Society of Intensive Care Medicine, 40 Avenue Joseph Wybran, 1070 Brussels, Belgium

e-mail: public@esicm.org

The following abstracts have been submitted through the Internet for the European Society of Intensive Care Medicine annual congress in Amsterdam in October 2003. Unfortunately, due to imprecise instructions, the list of the co-authors has been published incompletely. Here are the correct abstract co-authors.

\section{Intensive Care Med, Supplement 1 (2003) 29: S19-S63}

199 PERCU TWIST TRACHEOSTOMY:

FIRST EXPERIENCE IN A NEUROSURGICAL ICU

Moretti $\mathrm{M}^{1}$, Magenta $\mathrm{P}^{1}$, Arghetti $\mathrm{S}^{1}$, Levati $\mathrm{A}^{1}$, Vesconi $\mathrm{S}^{1}$

${ }^{1}$ Neur ICU, AO Ospedale Niguarda Cà Granda, Milano, Italy

\section{Intensive Care Med, Supplement 1 (2003) 29: S79-S122}

378 PREVALENCE AND MOLECULAR TYPING

OF ACINETOBACTER BAUMANNII

IN AN ICU-PRELIMINARY RESULTS

Paramythiotou $\mathrm{E}^{1}$, Mylona-Petropoulou $\mathrm{D}^{2}$, Vagiakou-Voudri $\mathrm{H}^{2}$, Tassios $\mathrm{PT}^{3}$, Tzouvelekis $\mathrm{LS}^{3}$, Legakis $\mathrm{NJ}^{3}$, Malamou-Lada $\mathrm{H}^{1}$, Karabinis $\mathrm{A}^{1}$

${ }^{1} \mathrm{ICU}$

${ }^{2}$ Microbiology Department, G.Gennimatas Hospital

${ }^{3}$ Department of Microbiology, Medical School,

National and Kapodestrian University, Athens, Greece

382 VANCOMYCIN-RESISTANT ENTEROCOCCI

IN AN INTENSIVE CARE UNIT

Paramythiotou $\mathrm{E}^{1}$, Kontopidou $\mathrm{F}^{1}$, Mylona-Petropoulou $\mathrm{D}^{2}$, Kalogeromitros $\mathrm{A}^{1}$, Poularas $\mathrm{I}^{1}$, Krikos $\mathrm{V}^{1}$, Ntagiopoulos $\mathrm{P}^{1}$, Katsarellis $\mathrm{N}^{1}$, Karabinis $\mathrm{A}^{1}$

${ }^{1} \mathrm{ICU}$

${ }^{2}$ Microbiology Department, G. Gennimatas Hospital, Athens, Greece

458 NURSING DEPENDENCY SCORING SYSTEM: A LOCAL ASSESSMENT

Bolger $\mathrm{R}^{1}$, Ware $\mathrm{M}^{1}$, Smithies $\mathrm{M}^{1}$

${ }^{1}$ Critical Care Directorate, Cardiff and Vale NHS Trust, Cardiff, United Kingdom

\section{Intensive Care Med, Supplement 1 (2003) 29: S123-S145}

488 STRESS ULCER PROPHYLAXIS (SUP):

PRESCRIPTION ATTITUDES IN ICU

AND INFLUENCE ON THE INCIDENCE OF VAP

Arvaniti K, Liatsi D, Tsagourias M, Synnefaki E, Matamis D

${ }^{1}$ Intensive Care Unit, Papageorgiou General Hospital,

Thessaloniki, Greece 